\title{
Antigen-specific immune responses to influenza vaccine in utero
}

\author{
Deepa Rastogi,, ${ }^{1}$ Chaodong Wang, ${ }^{2}$ Xia Mao, ${ }^{3}$ Cynthia Lendor, ${ }^{3}$ \\ Paul B. Rothman, ${ }^{4}$ and Rachel L. Miller ${ }^{3}$
}

\begin{abstract}
${ }^{1}$ Children's Hospital at Montefiore, Albert Einstein College of Medicine, New York, New York, USA. 2Department of Neurology, Jiangxi Provincial People's Hospital, Nanchang, People's Republic of China. ${ }^{3}$ Department of Medicine, Division of Pulmonary, Allergy, and Critical Care Medicine, Columbia University College of Physicians and Surgeons, New York, New York, USA. ${ }^{4}$ Department of Internal Medicine, University of lowa, lowa City, Iowa, USA.
\end{abstract}

\begin{abstract}
Initial immune responses to allergens may occur before birth, thereby modulating the subsequent development of atopy. This paradigm remains controversial, however, due to the inability to identify antigen-specific $T$ cells in cord blood. The advent of MHC tetramers has revolutionized the detection of antigen-specific T cells. Tetramer staining of cord blood after $\mathrm{CMV}$ infection has demonstrated that effective $\mathrm{CD8}^{+}$antigen-specific immune responses can follow intrauterine viral infections. We hypothesized that sensitization to antigens occurs in utero in humans. We studied cord blood $B$ and $T$ cell immune responses following vaccination against influenza during pregnancy. Anti-Fluzone and anti-matrix protein IgM antibodies were detected in $38.5 \%$ (27 of 70 ) and $40.0 \%$ (28 of 70), respectively, of cord blood specimens. Using MHC tetramers, HA-specific $\mathrm{CD}^{+}{ }^{+} \mathrm{T}$ cells were detected among $25.0 \%$ (3 of 12 ) and $42.9 \%$ (6 of 14$)$ of cord blood specimens possessing DRB $1 * 0101$ and DRB $1 * 0401$ HLA types, respectively, and were detected even when the DRB1 HLA type was inherited from the father. Matrix protein-specific $\mathrm{CD8}^{+} \mathrm{T}$ cells were detected among $10.0 \%$ ( 2 of 20$)$ of $\mathrm{HLA}-\mathrm{A}^{*} 0201^{+}$newborns. These results suggest that $B$ and $T$ cell immune responses occur in the fetus following vaccination against influenza and have important implications for determining when immune responses to environmental exposures begin.
\end{abstract}

\section{Introduction}

Fetal immune responses following exposure of the mother to allergens or other molecules during pregnancy may affect the risk for the subsequent development of atopy and other diseases. Support for this has been based on several observations. Allergen-induced cord blood mononuclear cell (CBMC) proliferative responses have been documented and found to be distinct from the mother's mononuclear cell proliferative responses (1-5). Altered cord blood cytokine levels, such as reduced IFN- $\gamma$, appear to be associated with the subsequent development of atopic dermatitis, allergic rhinitis, or asthma $(6,7)$. In a murine model, prenatal exposure to endotoxin downregulated allergic sensitization and airway inflammation in the offspring (8). Finally, maternal, but not paternal, IgE levels have been associated with infant IgE levels and later development of atopy (9).

Nonetheless, the fundamental paradigm that adaptive antigenspecific $T$ cell and B cell immune responses to environmental exposures occurs prenatally remains controversial $(10,11)$. The specificity of the cord blood proliferation has been challenged, as $\mathrm{T}$ cell epitope mapping of cord blood responses to allergen indicates that neonatal immune cells lack the fine specificity of adult memory cells (12). Also, CBMCs can proliferate following in vitro stimulation with non-recall antigens, to which natural exposure is extremely unlikely $(13,14)$.

The controversy surrounding in utero sensitization is not only due to the uncertainty regarding the specificity of proliferative immune responses but, importantly, stems from the inability to detect and immunophenotype antigen-specific $\mathrm{CD}^{+}{ }^{+}$and $\mathrm{CD} 8^{+} \mathrm{T}$ cells. Conventional techniques for quantifying antigen-specific T cells include limiting dilution cloning, ELISPOT, and intracellular cytokine assays.

Nonstandard abbreviations used: CBMC, cord blood mononuclear cells; GAD, glutamic acid decarboxylase; JE, Japanese encephalitis; MP, matrix protein. Conflict of interest: The authors have declared that no conflict of interest exists. Citation for this article: J. Clin. Invest. 117:1637-1646 (2007). doi:10.1172/JCI29466
Although these assays can be conducted without knowledge of specific antigen epitopes and/or the $\mathrm{MHC}$ restriction elements, they are indirect and prone to considerable experimental variability (15).

The advent of MHC class I and II multimer reagents has revolutionized the detection of antigen-specific $\mathrm{T}$ cells. The recognition is based on the specific trimolecular interaction between the MHCpeptide and the T cell receptor (16). MHC class I and II tetramers consist of 4 linked HLA molecules loaded with a peptide (epitope) such that the MHC-peptide complex can be recognized by a subset of specific T cells via the TCR. The tetramer molecule is covalently conjugated to a fluorochrome, allowing sensitive, direct, and specific detection of $\mathrm{CD}^{+}$or $\mathrm{CD}^{+} \mathrm{T}$ cells by flow cytometry $(16,17)$. Tetramer-based detection has been applied successfully to the investigation of cell-mediated immunity to a number of pathogens in peripheral blood from children and adults (17-19). Further, tetramers have been able to identify and immunophenotype antigen-specific T cells in cord blood following human CMV infection, demonstrating that effective $\mathrm{CD}^{+}$antigen-specific immune responses can occur following intrauterine viral infection (20).

Literature demonstrating the development of antigen-specific $B$ cells in utero has been scarce. In 2 small case series, maternal vaccination against tetanus was associated with the development of antitetanus IgM in the cord blood $(21,22)$. In another case series, influenza-specific IgM was measured in the cord blood of 1 of 8 babies born to vaccinated mothers (23). Details concerning the frequency of neonatal humoral immunity following vaccination and exposure to other antigens and the relationship between maternal and cord blood immunoglobulin responses have yet to be addressed.

We hypothesized that in utero sensitization occurs in response to antigens to which the mother is exposed during pregnancy. To address this hypothesis, and to study systemically the mechanism underlying in utero $\mathrm{T}$ cell and $\mathrm{B}$ cell immune responses in humans, we chose a model in which the pregnant mother's exposure to anti- 


\section{Table 1}

Characteristics of the fully enrolled study cohort $(n=126)$

$\begin{array}{lc}\text { Characteristic } & \text { Result } \\ \text { Ethnicity } & \\ \text { Hispanic } & 111 \\ \text { African American } & 13 \\ \text { White } & 2 \\ \text { Age at enrollment (yr, mean } \pm \text { SEM) } & 25.41 \pm 0.42 \\ \text { Gestation at vaccination (wk, mean } \pm \text { SEM) } & 29.53 \pm 0.34 \\ \text { HLA-A*0201+ } & 43.7 \% \\ \text { DRB1* } 0101^{+} & 17.5 \% \\ \text { DRB1 }^{+} 0401^{+} & 18.2 \%\end{array}$

gen is controllable, safe, and recommended by the Centers for Disease Control and Prevention (CDC) (24), as well as a model in which the detection of the fetal immune response to antigen is sensitive and specific. The strategy was to study cord blood B cell and T cell immune responses following vaccination of the pregnant mothers against influenza (Fluzone vaccine, zonal purified, subvirion). We found that the fetus readily produces anti-Fluzone and anti-matrix protein (anti-MP) IgM following vaccination of the mothers against influenza during pregnancy. HA-specific $\mathrm{CD}^{+} \mathrm{T}$ cells were detected in cord blood using tetramer staining of specimens from newborns who inherited the DRB1 HLA types from either the mother or father. Combined, these results suggest that intrauterine B and $\mathrm{T}$ cell immune responses occur following vaccination of the mother and support the paradigm that specific fetal immune response to environmental exposures occurs prenatally.

\section{Results}

The cohort consisted predominantly of Hispanic women, at least 52\% Dominican American, receiving the influenza vaccine at $29.53 \pm 0.34$ weeks gestation (Table 1 ). The frequency of HLA-A*0201 in the cohort is within a range reported by other groups (25-27), but the frequencies of DRB $1 * 0101$ and DRB1*0401 may be higher when compared with those in other Hispanic cohorts (27-29). Among newborns who inherited 1 of the 3 HLA types of interest, $57.6 \%$ (19 of 33), 15.4\% (2 of 13), and $44.4 \%$ (8 of 18 ) of them shared the $A^{*} 0201$, DRB $1 * 0401$, and DRB1*0101 HLA types, respectively, with their mothers.

$B$ cell responses to influenza vaccine among pregnant women and in cord blood. Maternal B cell immune responses to the influenza vaccine during pregnancy were determined by comparing prevaccination with postvaccination levels of anti-Fluzone and anti-MP IgM and IgG antibodies. Among the 89 mothers for whom both prevaccination and postvaccination specimens were available, 43.8\% (39 of 89) produced greater than 2-fold increases in IgM antibodies against Fluzone, a seroconversion rate modestly lower than that in previous reports following vaccination of pregnant women against influenza (30) and group B streptococcus (31). In contrast, 20.2\% (18 of 89) of mothers produced greater than 2-fold increases in levels of IgM antibodies against MP (Figure 1). Further analysis demonstrated that the mothers' overall mean postvaccination anti-Fluzone IgM antibody was significantly greater than the prevaccination level $(0.35 \pm 0.04$ versus $0.17 \pm 0.03 ; P<0.0001$; antibody levels expressed in OD units). Such overall increases following vaccination were not significant against $\mathrm{MP}(0.12 \pm 0.02$ versus $0.06 \pm 0.01 ; P=0.11)$. The likelihood of seroconversion was not affected by the time interval between pre- and postvaccination measurements, when groups of women vaccinated at 24-28, 28-32, and more than 32 weeks gestation were compared $(36 \%, 31.8 \%$, and $31.8 \% ; P=0.95)$. These results also indicate that anti-Fluzone IgM antibodies can be maintained for up to 16 weeks. In comparison, measured postvaccination antiFluzone and anti-MP IgG antibodies were increased among $13.5 \%$ (12 of 89 ) and $27 \%$ (24 of 89) of the pregnant mothers, respectively. The mean postvaccination anti-Fluzone, but not anti-MP, IgG antibody was significantly greater than the prevaccination level (Fluzone, $1.85 \pm 0.09$ versus $1.32 \pm 0.07, P<0.0001$; MP, $0.27 \pm 0.07$ versus $0.19 \pm 0.05, P=0.14$ ).

To determine whether antigen-specific $B$ cell responses to influenza antigens occur in utero, and to describe its frequency, anti-influenza IgM and IgG antibodies in cord blood were measured. Increased (i.e., ODs 2-fold greater than those among the "nonvaccinated" negative control cohort) anti-Fluzone and anti-MP IgM antibodies were measured among 38.6\% (27 of 70) and 40\% (28 of 70), respectively, of the cord blood specimens following vaccination of the mothers. Also, anti-Fluzone and anti-MP IgM mean antibodies were significantly greater in cord blood from mothers who received the influenza vaccine when compared with cord blood from newborns of unvaccinated mothers (Fluzone, $0.05 \pm 0.01$ versus $0.01 \pm 0.005$; MP, $0.03 \pm 0.01$ versus $0.006 \pm 0.003$, respectively; $P<0.05)$. Moreover, mean levels of cord blood anti-Fluzone and MP IgM antibodies were significantly higher than those of antibodies against an irrelevant antigen, Japanese encephalitis (JE) (Fluzone, $0.09 \pm 0.02$ and MP, $0.07 \pm 0.01$ versus JE, $0.01 \pm 0.004 ; P<0.001$ ) (Figure 1). As an additional negative control, cord blood anti-Fluzone and anti-MP IgM levels of newborns of unvaccinated mothers were found to be similar to cord blood anti-JE IgM levels of newborns
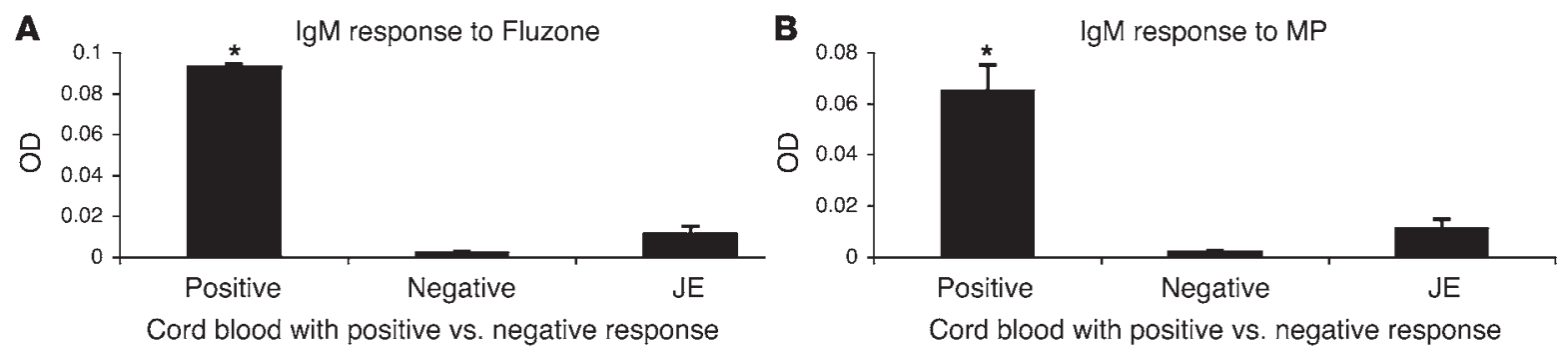

\section{Figure 1}

Anti-Fluzone and anti-MP OD ratios from cord blood following vaccination of the mothers. Positive and negative mean levels were each compared with the mean anti-JE antibody for both Fluzone (A) and MP (B). Among cord blood samples with positive anti-IgM antibodies, levels were significantly greater than those measured in response to JE. ${ }^{*} P<0.01$ versus JE. 


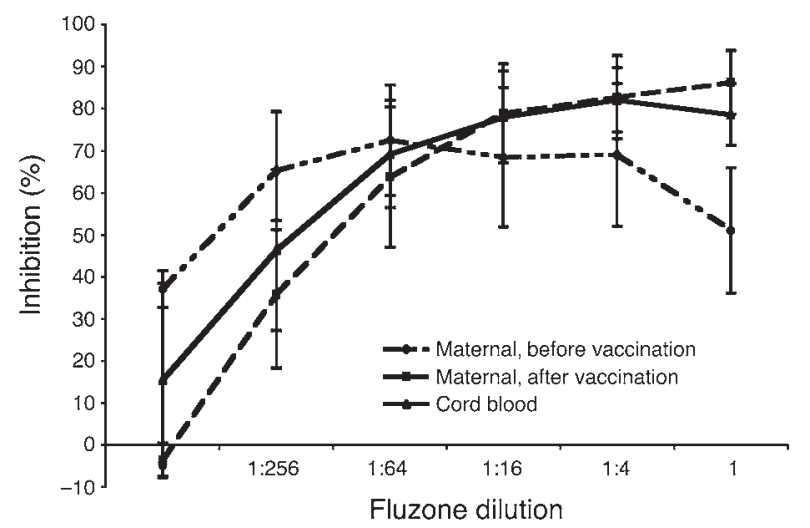

Figure 2

Results of Fluzone inhibition assay following vaccination of the mothers.

of mothers vaccinated against influenza. Cord blood anti-Fluzone and anti-MP IgG antibodies also tended to increase following vaccination of the mother during pregnancy (Fluzone, $2.09 \pm 0.1$ versus $1.71 \pm 0.12, P=0.05 ; \mathrm{MP}, 0.37 \pm 0.10$ versus $0.07 \pm 0.02, P<0.005$ ). But cord blood anti-Fluzone and anti-MP IgM antibodies did not correlate with their respective IgG antibodies (Fluzone, $r=-0.23$, $P=0.06$; MP, $r=-0.02, P=0.85)$. Combined, these results suggest that both anti-influenza IgM and IgG antibodies can be detected in the cord blood following vaccination of the mother during pregnancy, with the latter presumably developing in the cord blood as a result of receptor-mediated maternofetal transfer (32).

The specificity of anti-Fluzone IgM in maternal and cord blood was confirmed further by Fluzone inhibition assays. As shown in Figure 2, the percentage of Fluzone inhibition increased following preincubation with increasing concentrations of Fluzone vaccine (from $-3.6 \% \pm 4.1 \%$ to $86.2 \% \pm 7.6 \%$ maternal postvaccination positive samples; $15.5 \% \pm 22.9 \%$ to $78.6 \% \pm 7.4 \%$ in cord blood positive samples). Such inhibition was not found among the maternal prevaccination specimens.

The relationship between levels of anti-Fluzone and anti-MP IgM antibodies in maternal and cold blood was also examined. As shown in Figure 3, a modest correlation between maternal and cord blood anti-Fluzone $(r=0.26 ; P=0.027)$, but not anti-MP $(r=0.19 ; P=0.1)$, IgM levels was present. Interestingly, 63\% (17 of 27) of the positive anti-Fluzone IgM cord blood responses occurred in the absence of a positive response in the mother. Similarly, 67.8\% (19 of 28) of the positive anti-MP IgM cord blood responses occurred in the absence of a positive response from the mother (Table 2). In contrast, a very strong correlation was observed between the mothers' and cord blood anti-Fluzone $(r=0.94 ; P<0.0001)$ and anti-MP $(r=0.95 ; P<0.0001)$ IgG antibody levels (Figure 3), consis- tent with the known maternal-to-fetal placental passage of IgG. In sum, these results suggest that the fetal generation of anti-Fluzone and anti-MP IgM may be positively associated with, but still occur independently of, the maternal production of anti-Fluzone and anti-MP IgM antibodies following vaccination.

Identification of maternal and cord blood $\mathrm{HA}_{306-318-\text { specific }} \mathrm{CD}^{+} \mathrm{T}$ cells by direct staining with MHC class II tetramer. To measure the development of influenza-specific $\mathrm{T}$ cell responses following vaccination during pregnancy, the generation of maternal and cord blood $\mathrm{HA}_{306-318^{-}}$ specific $\mathrm{CD}^{+} \mathrm{T}$ cells was analyzed by direct staining with $\mathrm{MHC}$ class II tetramer using criteria for positivity as described in Methods. HA-specific $\mathrm{CD}^{+} \mathrm{T}$ cells were detected among $57.1 \%$ (4 of 7) of DRB $1 * 0101^{+}$vaccinated pregnant women and among $35.7 \%$ (5 of 14 ) of DRB ${ }^{*} 0401^{+}$vaccinated pregnant women using the respective HLA-matched tetramers. No tetramer staining was observed with these reagents among any of the DRB $1^{*} 0101^{-}$or DRB ${ }^{*} 0401^{-}$mothers $(n=20)$ tested. Among maternal specimens that were classified as demonstrating positive tetramer staining, $75 \%$ (6 of 8 ) of them showed a corresponding increase in tetramer staining compared with the prevaccination levels. In comparison, substantial tetramer staining was detected among $25.0 \%$ (3 of 12) and 42.9\% (6 of 14) of newborns who inherited the DRB1*0101 (Figure 4) and DRB1*0401 HLA types, respectively (Figure 5). DR1/HA or DR4/HA tetramer staining was not detected among any of the DRB $1^{*} 0101^{-}$or DRB $1 * 0401^{-}$ cord blood specimens $(n=11)$. The frequency of DR1/HA and DR4/HA tetramer positivity among $\mathrm{CD}^{+}$cord blood cells ranged $0.03 \%$ to $0.18 \%$ and $0.01 \%$ to $0.10 \%$, respectively.

To determine whether the simultaneous detection of antigenspecific $T$ cells in both maternal peripheral blood and cord blood represents contamination of maternal cells during the collection of cord blood, cord blood specimens from newborns who inherited the DRB $1 * 0101$ or DRB $1 * 0401$ HLA type from the father were examined. Among the 9 newborns with evidence of increased DR1/HA or DR4/HA tetramer staining, 5 inherited the HLA type from the father (Figure 5). These data argue against the possibil-
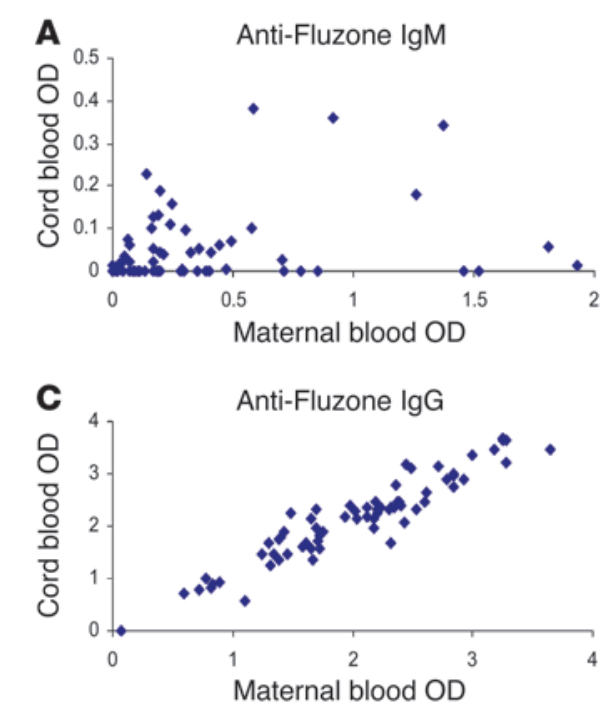

Figure 3

Comparison of ODs. (A) Comparison between maternal and cord blood anti-Fluzone lgM. $r=0.26$; $P=0.027$. (B) Comparison between maternal and cord blood anti-MP IgM. $r=0.19 ; P=0.1$. (C) Comparison between maternal and cord blood anti-Fluzone IgG. $r=0.94 ; P<0.0001$. (D) Comparison between maternal and cord blood anti-MP IgG. $r=0.95 ; P<0.0001$. 


\section{Table 2}

Discordance in anti-Fluzone and anti-MP IgM immune responses between maternal and cord blood

\begin{tabular}{l|lcc}
\multicolumn{1}{c}{ Maternal blood } \\
Cord blood & $\begin{array}{l}\text { Fluzone } \\
+\lg M\end{array}$ & 10 & $-\lg M$ \\
$-\lg M$ & 30 & $17^{\mathrm{A}}$ \\
$\operatorname{MP}$ & & 12 \\
$+\lg M$ & 9 & $19 \mathrm{~A}$ \\
$-\lg M$ & 10 & 41 \\
\hline
\end{tabular}

${ }^{A}$ Cord blood developed IgM even in the absence of mother's IgM.

ity that tetramer positivity of cord blood specimens during flow cytometry represents contamination by maternal cells.

Identification of maternal and cord blood MP-specific $C D 8^{+} T$ cells by direct staining with MHC class I tetramer. Relatively little is known about the $\mathrm{CD}^{+} \mathrm{T}$ cell response to influenza vaccination during pregnancy as measured in either maternal or cord blood. Notably, neonatal $\mathrm{CD}^{+} \mathrm{T}$ cell responses have been described as weak compared with those of adults (33) but perhaps more competent than previously recognized $(20,34)$. To help characterize these responses, we determined both maternal and cord blood influenza-specific $\mathrm{CD}^{+} \mathrm{T}$ cell responses following vaccination of the mother during pregnancy by immunostaining with MHC class I tetramer (A2.1/MP tetramer). Tetramer staining was detected only among $25 \%$ (4 of 16) of HLA-A $* 0201^{+}$mothers following vaccination and was not detected among any of the HLA-A*0201- $(n=14)$ specimens. Positive tetramer staining was present among only $10 \%$ ( 2 of 20 ) of HLA-A* $0201^{+}$ newborns tested and none $(n=13)$ of the HLA-A*0201- newborns tested (Figure 6). These results suggest that $\mathrm{CD}^{+} \mathrm{MP}$ tetramer positivity following vaccination during pregnancy is infrequent among vaccinated mothers and even less frequent among their newborns.

$H A$-specific $T$ cell responders may be effector memory $T$ cells. The demonstration of both humoral and cellular responses to influenza antigens in cord blood lends support to the notion that the fetus is capable of developing adaptive immunity to antigens encountered in utero. Such immunity would be expected to result in immunological memory in the newborn. To determine whether the HA tetramerpositive $\mathrm{CD}^{+} \mathrm{T}$ cells were of an effector memory phenotype, we per- formed additional costaining with CD45RO. As shown in Figure 7, the majority of maternal DR1/HA, DR4/HA tetramer-positive CD4+

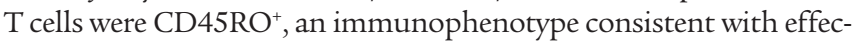
tor memory. To determine whether tetramer positivity in cord blood is associated with a memory phenotype, cord blood samples were stained for CD45RO cell surface expression. Convincing evidence of CD45RO expression (beyond that stained with the isotype staining control) was present among CD4 ${ }^{+} \mathrm{DR} 4 / \mathrm{HA}^{+}$cord blood cells $(66.7 \%$ and $14.1 \%$ of $\mathrm{CD}^{+}{ }^{+} \mathrm{DR} 4 / \mathrm{HA}^{+}$cells versus $6 \%$ and $0 \%$ of $\mathrm{CD}^{+} \mathrm{DR} 4 /$ glutamic acid decarboxylase $\left(\mathrm{CD} 4^{+} \mathrm{DR} 4 / \mathrm{GAD}^{+}\right)$cells in 2 consecutive experiments), consistent with an effector memory phenotype.

In vitro expansion of tetramer-positive T cells. To determine whether tetramer-positive $\mathrm{T}$ cells expand following in vitro stimulation with influenza vaccine, in vitro proliferation in thawed aliquots of available DR1, DR4/HA tetramer-positive and -negative cord blood specimens were tested using the cell tracking dye $\operatorname{CSFE}(35,36)$. In vitro proliferation followed by positive tetramer staining of $\mathrm{CD} 4^{+}$cells was limited and nonsustainable beyond 2-3 generations of divisions. Enrichment of tetramer staining was minimal (data not shown).

Influenza antigen-specific CBMC proliferation was infrequent and discordant with tetramer staining. To assess the in vitro proliferative response against influenza in maternal versus cord blood, and to compare influenza-specific $\mathrm{T}$ cell proliferation with tetramer staining and $\mathrm{B}$ cell responses, Fluzone-specific PBMC and CBMC proliferative responses were measured. Among the vaccinated mothers examined, 78\% (32 of 46) exhibited 2-fold increases in proliferation following in vitro stimulation with Fluzone, compared with proliferation in the absence of antigens. In comparison, $13.6 \%$ (6 of 44) of CBMC specimens exhibited 2-fold increases in proliferation following stimulation with Fluzone, compared with background proliferation. Notably, no association between the presence of influenza vaccine-induced proliferation and detection of $\mathrm{CD}^{+}-\mathrm{HA}$-specific cells by tetramer staining was found (Table 3). Likewise, positive results from either $\mathrm{T}$ cell assay were not associated with greater levels of IgM antibodies (Table 3).

\section{Discussion}

The determination that the fetus can initiate $\mathrm{B}$ and $\mathrm{T}$ cell responses following exposure of the mother during pregnancy has wide implications for multiple diseases, including atopy. This study provides the first direct evidence of antigen-specific $\mathrm{T}$ cell fetal immune responses to prenatal antigen exposure via vaccination. Without in vitro manipulation, MHC class II HA-specific $\mathrm{CD}^{+} \mathrm{T}$ cells were detected in $34.6 \%$ ( 9 of 26) of the cord blood samples. MHC class I
Figure 4

Representative tetramer staining of DRB $1^{*} 0101^{+}$cord blood stained with the DR1/HA tetramer (A), the same DRB $1{ }^{*} 0101^{+}$cord blood stained with the DR4/HA negative control tetramer (B), and a sample from a DRB ${ }^{*} 0101^{-}$adult volunteer stained with the DR1/HA tetramer $(\mathbf{C})$. Panels show percentage of tetramer-staining-positive cells from a representative subject.
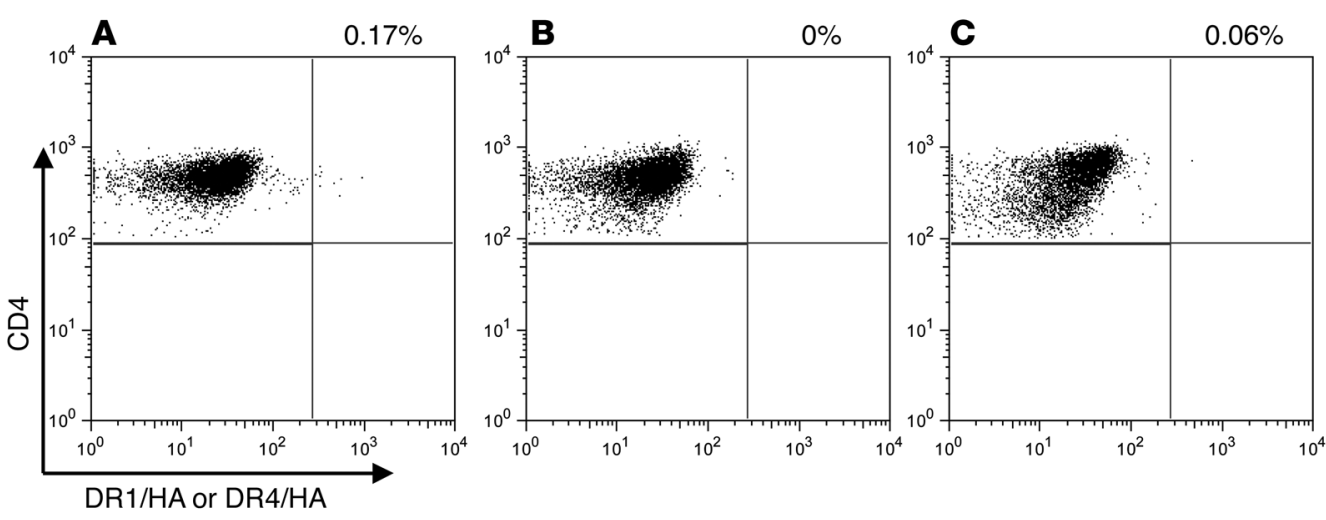


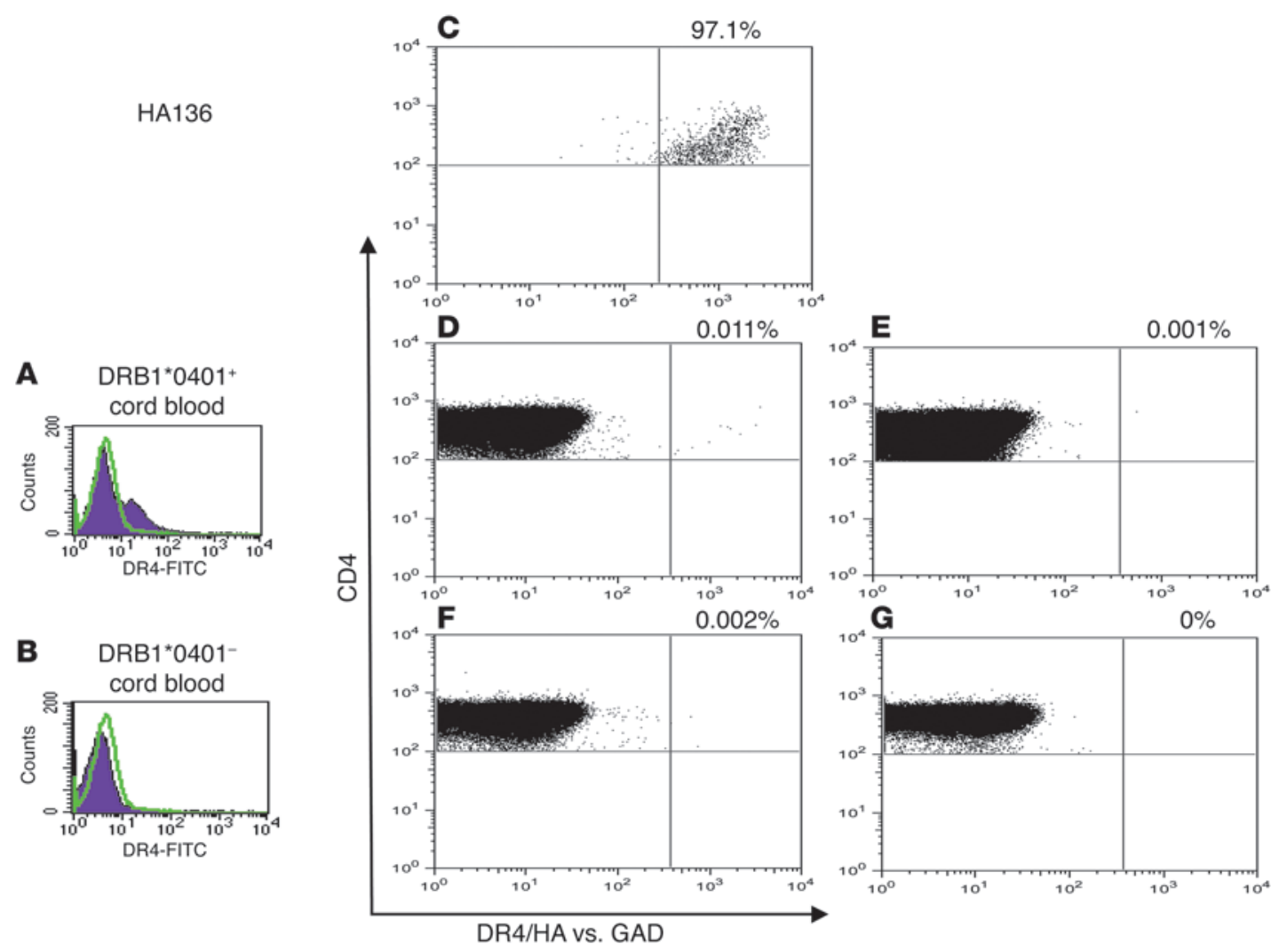

Figure 5

Representative comparison of tetramer staining in DRB1*0401+ and DRB1 $0401^{-}$cord blood. (A and B) DR4 HLA typing by flow cytometry. (C) DRB1*0401+-HA T cell clone (HA136). (D) DRB1*0401+ inherited from father; staining performed with DR4/HA tetramer. (E) DRB1*0401+ inherited from father; staining performed with DR4/GAD tetramer. (F) DRB1*0401- newborn; staining performed with DR4/HA tetramer. (G) DRB1*0401newborn; staining performed with DR4/GAD tetramer. Panels show percentage of tetramer-staining-positive cells from a representative subject.

MP-specific CD8 ${ }^{+} \mathrm{T}$ cells were detected less frequently. In addition, influenza-specific B cell responses in cord blood, as assessed by IgM production following vaccination of the mother were relatively common (38.5\% to Fluzone; $40 \%$ to MP). Combined, these results suggest that $\mathrm{T}$ and $\mathrm{B}$ cell immune responses occur in the fetus following maternal vaccination against influenza during pregnancy.

MHC tetramers utilize the MHC-peptide complex to detect antigen-specific $T$ cells at the single-cell level, thus surpassing the detection limits of conventional cell-based methods. Lower detection limits for the tetramers utilized here have been reported. For example, Dunbar and colleagues reported that the A2.1/MP tetramer stained $0.0017 \%-0.067 \%$ of PBMCs from HLA A* $0201^{+}$individuals with no known history of influenza infection to demonstrate that tetramers could be used to analyze low-frequency $\mathrm{CD}^{+}$ populations (17). Hoffmann and colleagues reported that $0.11 \%$ of HLA A* $0201^{+} \mathrm{CD}^{+}$cells collected from healthy donors stain with the A2.1/MP tetramer (37). The latter group also calculated a geometric mean background staining among HLA $A^{*} 0201^{-}$subjects of $0.0045 \%$. They also established a cutoff for the lower detection limit of the assay at the upper 99th percentile of tetramer-positive $\mathrm{CD}^{+} \mathrm{T}$ cells in HLA A*0201 individuals at $0.01 \%$ (37), providing guidelines for discriminating true positive events from false negative ones. Tetramer staining also detected $0.13 \%-7.5 \% \mathrm{CD}^{+}$cells from CMV-infected cord blood but repeatedly less than $0.01 \%$ of $\mathrm{CD}^{+}$cells from noninfected cord blood (20). When examining
$\mathrm{CD}^{+}$immune responses following vaccination against influenza, Danke and Kwok reported that $0.0033 \%-0.167 \%$ of $\mathrm{CD}^{+}{ }^{+}$cells stain with the DR1/HA or DR4/HA tetramer (38), though expected background tetramer staining among DRB ${ }^{*} 0101^{-}$or DRB ${ }^{*} 0401^{-}$or nonvaccinated subjects has not been well described. While potential overlap tetramer staining between true antigen-specific cells and antigen-nonspecific, falsely positive cells cannot be ignored, the frequency of DR1/HA, DR4/HA CD4+ positivity among specimens classified as positive here $(0.01 \%-0.18 \%$, after subtracting measured background levels) ranges above reported background levels. Confirmation of antigen specificity during the measured immune responses was achieved by: (a) excluding the possibility of contamination from maternal cells by detecting cord blood DR1/HA and DR4/HA tetramer staining among newborns who inherited the HLA type from their fathers; (b) use of multiple negative controls, including a tetramer against an unrelated antigen and DR1/HA or DR4/HA- vaccinated adult and cord blood samples; and (c) demonstration of increased tetramer staining in postvaccination compared with prevaccination maternal specimens (Figure 7) and among 75\% (6 of 8) of tested specimens.

In contrast to $\mathrm{HA}$-specific $\mathrm{CD}^{+} \mathrm{T}$ cells, $\mathrm{MP}$-specific $\mathrm{CD}^{+} \mathrm{T}$ cells following vaccination were difficult to detect in maternal samples. This finding is contrary to documentation of MP-specific CD8 ${ }^{+}$ $\mathrm{T}$ cells following influenza vaccination among healthy, young, nonpregnant individuals $(39,40)$. Likewise, the development of 

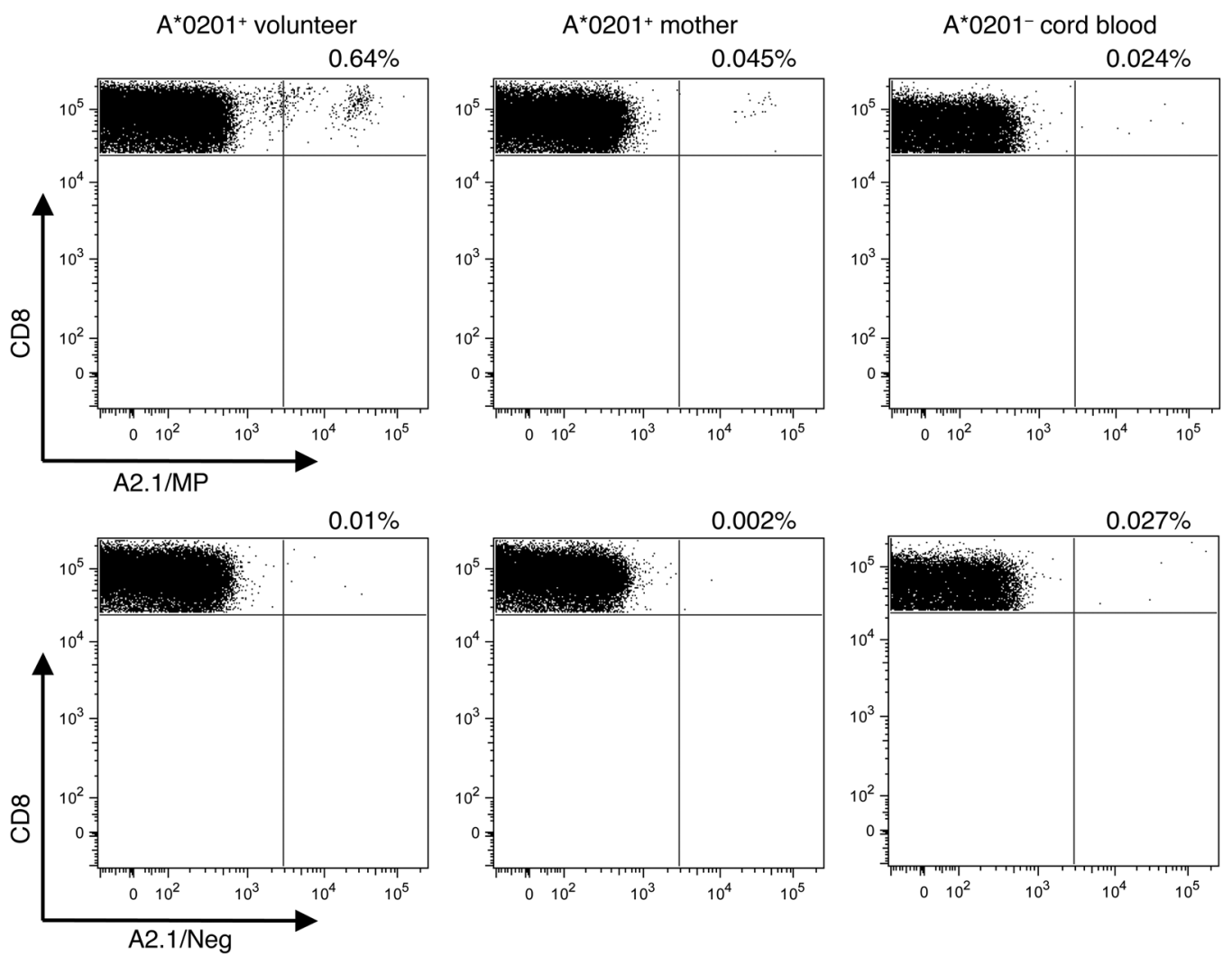

Figure 6

Representative tetramer staining of samples from an HLA A*0201+ adult volunteer following influenza vaccination; an HLA A*0201+ mother after vaccination against influenza; and cord blood from her HLA A*0201- newborn. Bottom row represents staining using a negative control tetramer (A2.1/Neg). The cord blood specimen was classified as negative. Panels show percentage of tetramer-staining-positive cells from a representative subject.
MP-specific $\mathrm{CD}^{+} \mathrm{T}$ cells following vaccination of the mother was extremely difficult to detect in cord blood samples, despite the observation that $\mathrm{CMV}$-specific $\mathrm{CD}^{+}$cord cells staining positive for tetramer are readily detectable following CMV infection (20). The findings here suggest that the predominant fetal immune responses to vaccination are $\mathrm{MHC}$ class II and B cell mediated. The mechanism of this apparent $\mathrm{CD}^{+}$-dominated response to influenza vaccine in the fetus is unclear, but the response has been observed after vaccination against influenza of children following stem cell transplants and among the elderly (40-42).

Cord blood $\mathrm{T}$ cells have been characterized as predominantly naive cells, in part because more than $90 \%$ of circulating $\mathrm{CD}^{+} \mathrm{T}$ cells are $\mathrm{CD}_{45 \mathrm{RO}^{-}}(43,44)$. Nevertheless, CMV tetramer-positive $\mathrm{CD}^{+}$cells with increased CD45RO expression and cytolytic activity were detected in cord blood cells following CMV infection, indicating that neonatal cells can express a mature, antigen-specific phenotype (20). Similarly, in these experiments, influenza-specific cord blood $\mathrm{T}$ cells identified by specific tetramer staining (but not

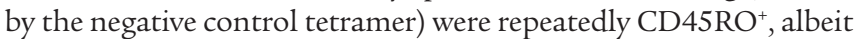
to a lesser degree than maternal PBMCs. This phenotype is not consistent with recent thymic emigrants and suggests successful generation of an effector memory $\mathrm{T}$ cell response.

This study also corroborates the findings and greatly supports the suggestion of previous reports that antigen-specific IgM antibodies develop in utero in response to vaccinations. The frequently higher level of influenza-specific IgM antibodies among newborns born to vaccinated mothers than those born to nonvaccinated mothers, and the absence of cord blood IgM antibodies against JE, both suggest that antigen-specific B cell immune responses to influenza vaccine occur in utero. The discordance between the newborns' and mothers' IgM seroconversion indicates that production of IgM in cord blood is independent of the mother's production of IgM.

Some reports suggest that maternally derived antibodies may inhibit offspring antibody production during infancy. These include studies following vaccination against measles (45) and hepatitis A (46) but not herpes (47). Persistent B cell inhibitory effects of maternal vaccination against influenza among the offspring was demonstrated elegantly by measuring maternally derived versus offspring-generated IgG antibodies using allotype markers (48). In these experiments, production of maternally derived antibodies did not affect the cell-mediated responses. Maternally derived antiinfluenza antibodies did suppress the ability of infant mice to generate their own serum antibody production but not their ability to mount a secondary antibody response to influenza infection (49). In comparison, the modest positive correlations between maternally derived and cord blood IgM antibodies reported here argue against an inhibitory effect of anti-influenza maternal antibodies generated during human pregnancy on cord blood production. However, the offspring's ability to mount a secondary response following reexposure to influenza antigens was not examined.

Influenza-specific IgM antibody production was not necessarily associated with a greater likelihood of positive DR1/HA, DR4/HA tetramer staining in either the maternal or cord blood specimens. The detection of tetramer staining also occurred discordantly with influenza-induced T cell proliferation. One may predict that the sensitivity of tetramer staining would be greater than the antigen-specific proliferation assay, due to its ability to identify antigen-specific single cells and minimize the effects of nonspecific background proliferation. But the $\mathrm{CD}^{+}$response following vaccination against influenza is directed against multiple epitopes, and an assay such as tetramer 


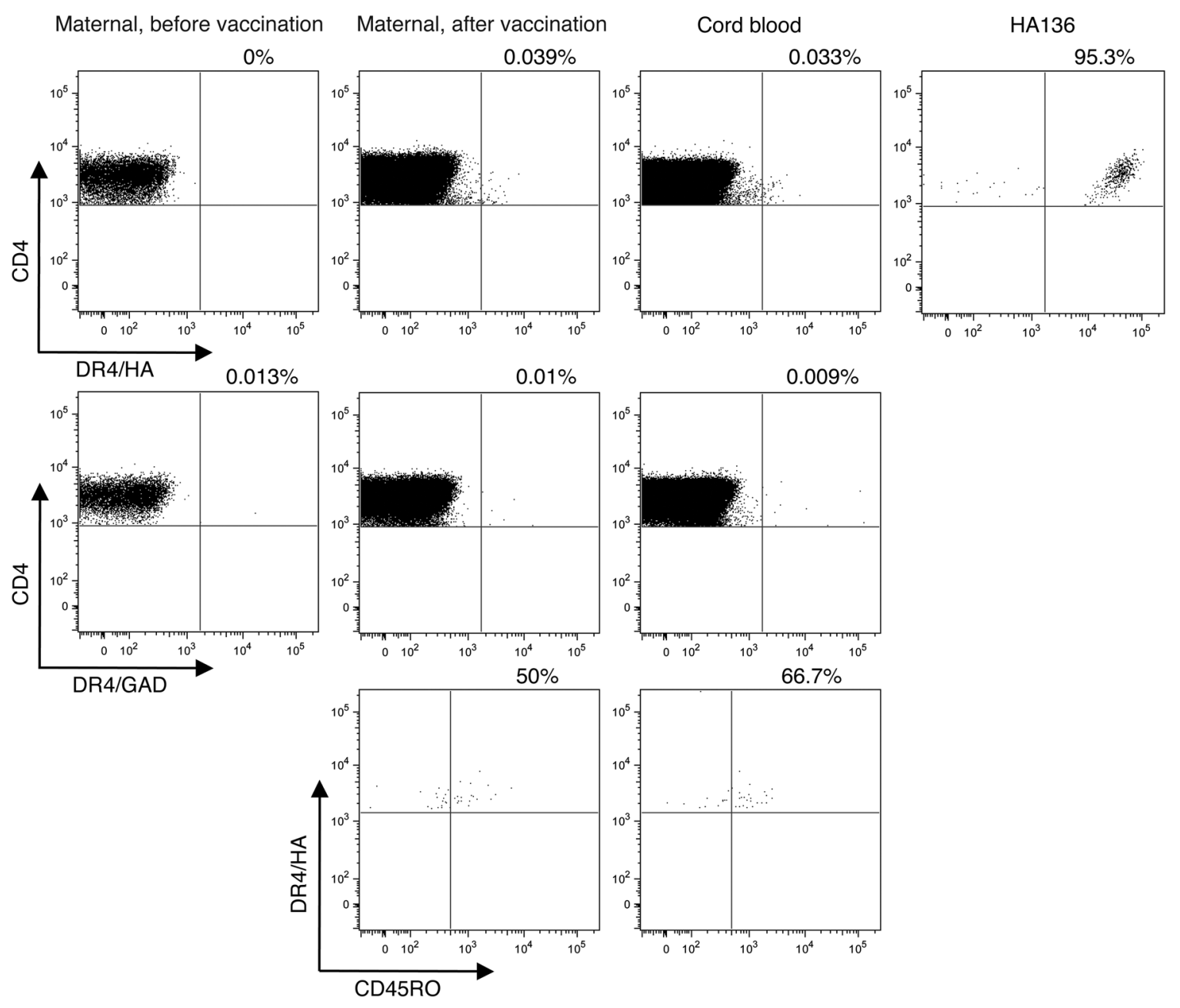

\section{Figure 7}

Maternal and neonatal influenza-specific CD4+ $T$ cells are CD45RO positive. Representative maternal PBMCs and CBMCs were stained with DR4/HA tetramers and anti-CD45RO antibody versus isotype control (data for the latter not shown). CD4+/DR4/HA ${ }^{+} \mathrm{T}$ cell population was gated to quantify the CD45RO ${ }^{+} \mathrm{T}$ cells. Background staining of postpartum maternal HLA DRB ${ }^{*} 0401^{-}$PBMCs was $0.008 \%$. Bottom panel indicates that maternal PBMCs and newborn CBMCs that stained with DR4/HA tetramer also expressed CD45RO $(50 \%$ and $66.7 \%$ DR4/HA+ cells, respectively). Panels show percentage of tetramer-staining-positive cells from a representative subject.

staining that measures the immune response against only one epitope will be less robust (38). The reduced proliferation rate observed among CBMCs when compared with PBMCs could also reflect differences in epitopes recognized by cord versus maternal cells.

There are certain limitations that may diminish the generalizability of these results. These include our inability to control completely for winter season (i.e., season for influenza infection) when making comparisons between the cohort born to vaccinated mothers and the cohort born to unvaccinated mothers. The $\mathrm{H} 3$ virus strains used in the influenza vaccines possess the PRYVKQNTLKLAT amino acid sequence, which differs from the tetramer epitope by 1 amino acid (i.e., PKYVKQNTLKLAT). The significance of this difference when tracking immune responses to vaccination is unclear. Despite the high accuracy of our HLA typing strategy, a small percentage could have been misclassified because sequencing data were not obtained in every case. In addition, direct detection of very infrequent events always raises concerns, especially when distinguishing small differences between antigen-specific staining and background staining. Collection of a large number of cells during flow cytometry $\left(3 \times 10^{5}\right.$ to $\left.1 \times 10^{6}\right)$ and use of appropriate and multiple controls help militate against effects of this problem. Moreover, tetramer staining can only analyze known MHC-specificities, however more complex epitopes may be targeted by $\mathrm{T}$ cells (50). We did not observe substantial enrichment of tetramer-positive cord blood cells following in vitro proliferation and tracking with CFSE, possibly consistent with the observation that CBMCs are characteristically more apoptotic than adult PBMCs and that the antigen-induced $\mathrm{T}$ cell apoptosis in CBMCs dominates the cell population under culture for 48-72 hours $(11,43)$. Finally, determination of the presence of antigen-specific $\mathrm{T}$ cells several months after birth using repeated tetramer staining may have provided further proof of the development of memory $\mathrm{T}$ cells, but such phlebotomy was beyond the scope of this study.

In summary, this study establishes that both $\mathrm{T}$ and $\mathrm{B}$ cell immune responses to antigens occur in utero following vaccination and these responses display features of immunological effector memory, a hallmark of adaptive immunity. This study 


\section{Table 3}

Comparison of cord blood tetramer staining with anti-influenza IgM production and T cell proliferation

\begin{tabular}{ccccc} 
& Tetramer result & Anti-Fluzone IgM & Anti-MP IgM & Fluzone proliferation \\
DRB1 ${ }^{*} 0101+$ or DRB1 ${ }^{*} 0401+$ & Positive $(n=9)$ & $33.3 \%(2 / 6)$ & $66.7 \%(4 / 6)$ & $0 \%(0 / 4)$ \\
& Negative $(n=17)$ & $37.5 \%(6 / 16)$ & $25.0 \%(4 / 16)$ & $22.2 \%(2 / 9)$ \\
\hline
\end{tabular}

supports the theory that the human neonatal immune system is not deficient or incompetent but, rather, capable of responding to environmental exposures. It remains unclear whether these results can be generalized to MHC class II immune responses to other environmental exposures, such as inhaled antigens, or whether neonatal priming may shape the subsequent postnatal vaccine or clinical response. Nonetheless, these results indicate that $\mathrm{B}$ and $\mathrm{T}$ cell immune responses occur in the fetus following vaccination against influenza and have important implications for determining when immune responses to environmental exposures begin.

\section{Methods}

Subject recruitment, influenza vaccination, and sample collection. 177 pregnant women were screened from prenatal clinics affiliated with New York Presbyterian Hospital. Inclusion criteria included expected delivery at New York Presbyterian Hospital or the affiliated Allen Pavilion during the season when influenza is most prevalent and age greater than 18 years. Exclusion criteria included: (a) history of cardiovascular, neurological, and other systemic disorders; (b) major complications during pregnancy or history of major complications during previous pregnancy; (c) history of recent HIV, $\mathrm{HBV}$, and other systemic infection; (d) history of allergic reaction to influenza and other vaccines, egg, and latex; and (e) recent flu-like symptoms. The study was approved by the Columbia University Institutional Review Board (IRB), and written informed consent was obtained from all participants. In 24 cases, the fathers of the expected newborn were recruited and HLA typing conducted following receipt of permission from the pregnant mothers followed by informed consent from the fathers as described below.

Influenza vaccine was administered to subjects during the second or third trimester up to 34 weeks gestation according to CDC recommendations. Of the 177 subjects screened, 126 were fully enrolled and received the influenza vaccination (123 Fluzone, 3 FluShield) (Sanofi Pasteur). The other 51 withdrew prior to vaccination due to inability to contact, voluntary withdrawal by the participant, onset of influenza infection, or administration of influenza vaccination prior to scheduled visit. The presence of HA and MP in the influenza vaccine was confirmed by Western blot analysis using $4 \mu \mathrm{g}$ of influenza vaccine and JE vaccine (negative control) and labeling with anti-HA (1:2,500) (Roche Applied Science) and anti-MP (1:2,500) (Accurate Chemical \& Scientific Corp.) antibodies (data not shown).

At the time of delivery, 87 adequate cord blood samples were collected successfully. The remaining 39 were not collected due to failure of the participant to contact research staff during labor, emergent delivery prohibiting timely collection of specimen, technical problems with the collection procedure, or delivery at home or another hospital. In cases of uncollected cord blood, collection of the maternal blood samples was deferred. Sera were not isolated from another $n=15$ of these cord blood samples due to technical problems (e.g., heparin contamination, inadequate amount collected).

Preparation of blood samples. Phlebotomy was performed on the mothers at recruitment, immediately prior to administration of the vaccine, and within 1 day postpartum. Cord blood was collected at delivery, as previously described (5). Sera were isolated from clotted blood, aliquoted, and frozen for future use, as described below. Maternal PBMCs and CBMCs were sepa- rated by density centrifugation from whole blood (5). Cells were counted and plated for culture (as described below) or frozen at $-80^{\circ} \mathrm{C}$ using freezing medium (10\% DMSO and $90 \%$ human serum). Additional samples $(n=42)$ of cord blood sera were collected from an unrelated cohort of children born to mothers who did not receive the influenza vaccine during pregnancy, usually because they were pregnant outside of the influenza season, using identical techniques and used as a negative control for ELISAs (described below).

ELISA. Anti-MP and anti-Fluzone IgM and IgG antibodies were measured in duplicate by ELISA. Prior to use, the influenza vaccine (Fluzone) and the JE vaccine were dialyzed for 36 hours using 3 exchanges of PBS and 10,000 MW cutoff (MWCO) dialysis tube (Pierce). Plates were coated with MP (80 ng/ml) (Doris Bucher, New York Medical College, Valhalla, New York, USA), dialyzed Fluzone vaccine (100 ng/ml), dialyzed JE vaccine ( $100 \mathrm{ng} / \mathrm{ml}$ ) (Sanofi Pasteur), or no antigen overnight. Unbound antigens were washed with PBS $/ 0.1 \%$ Tween. To block irrelevant proteins, $200 \mu$ l of blocking solution (1\% pig gelatin [Sigma-Aldrich] in PBS/0.1\% Tween) was added to each well and incubated for 2 hours at $37^{\circ} \mathrm{C}$. Sera in appropriate dilution (1:5,000 for IgM; 1:2,500 to $1: 15,000$ for IgG, usually $1: 10,000$ ) were added and incubated at $4^{\circ} \mathrm{C}$ for overnight. After incubation, wells were washed again with PBS/Tween buffer, and $100 \mu \mathrm{l} /$ well HRP-labeled goat-anti-human IgG or IgM (BioSource) was added and incubated at $37^{\circ} \mathrm{C}$ for 2 hours. In the final substrate reaction, $100 \mu \mathrm{l}$ of $3,3^{\prime}, 5,5^{\prime}$ tetramethylbenzidine (TMB) substrate (BD Biosciences - Pharmingen) was added to each well, samples were incubated in room temperature for 20 minutes, and the reaction was stopped by $1 \mathrm{M}$ phosphoric acid. ODs were read at $450 \mathrm{~nm}$ with a Molecular Devices plate reader.

The Fluzone inhibition assay was performed on select samples by preincubating serum samples prior to ELISA with serially diluted Fluzone vaccine (dilutions: 1:1, 1:4, 1:16, 1:64, 1:256) as well as a diluent alone (control) for 5 hours at $37^{\circ} \mathrm{C}$.

DNA extraction and HLA genotyping. After harvesting, mononuclear cells were incubated with lysis buffer (50 mM Tris, $100 \mathrm{mM}$ EDTA, $0.5 \%$ SDS) in the presence of proteinase $\mathrm{K}\left(1: 15\right.$ dilution) (Sigma-Aldrich) at $55^{\circ} \mathrm{C}$ overnight. DNA was isolated from CBMCs or PBMCs using phenol sevag extraction and alcohol precipitation according to conventional techniques (51).

HLA-A*0201, DRB $1 * 0101$, or DRB1*0401 genotypes were first screened in mothers' PBMCs and newborns' CBMCs by nested PCR, using the human HLA allele-specific primers, as described previously $(52,53)$, and reverse dot-blot hybridization, using allelic sequence-specific high-resolution oligonucleotides (Tepnel Lifecodes) as described previously (54). The nested PCR for HLA-A*0201, based on reports from Krausa and Browning, amplifies products that are $A^{*} 0201, A^{*} 0204, A^{*} 0207, A^{*} 0209, A^{*} 0211$, $A^{*} 02115 \mathrm{~N}, A^{*} 0216, A^{*} 0217$ with high resolution (55). Combined with high-resolution oligotyping (Tepnel Lifecodes) to eliminate the presence of HLA- $A^{*} 0204$ and $-A^{*} 0211$, the remaining subset of $\mathrm{A}^{+} \mathrm{A}^{*} 0201^{-}$individuals $\left(A^{*} 0207, A^{*} 0209, A^{*} 02115 \mathrm{~N}, A^{*} 0216, A^{*} 0217\right)$ is estimated to account for approximately $2 \%$ of all $\mathrm{A} 2{ }^{+}$Hispanic individuals tested (27), making misclassification unlikely. The reliability of this approach was confirmed through sequencing experiments of representative $(n=28)$ samples. Additional HLA typing was performed by flow cytometry using anti-HLA-A2 at 1:10 dilution (One Lambda Inc.). Equivocal DRB1*0401 results were confirmed by flow cytometry using an anti-DR4 antibody (Accurate). 
Tetramer staining and flow cytometry. The 4-color tetramer experiments were performed with MHC class I and II tetramer as described previously (38). In each of the experiments, $1 \times 10^{6}$ fresh or thawed PBMCs (mother's pre-vaccine and postpartum sample) or CBMCs were placed in $50 \mu \mathrm{l}$ of medium (RPMI medium [Mediatech Inc.] with $15 \%$ human serum) in a U-bottom, 96-well plate and blocked at $4^{\circ} \mathrm{C}$ for 30 minutes. After blocking, tetramer staining was conducted as follows: (a) MHC class I tetramer staining: $5 \mu$ l of PE-conjugated A2/M1 tetramer (HLA-A*0201/influenza $\mathrm{MP}_{58-66}$ peptide [GILGFVFTL]; iTag; Beckman Coulter) or negative control tetramer (PE-conjugated HLA-A*0201 unrelated, undisclosed peptide; Beckman Coulter) was added and incubated at room temperature for 30 minutes; (b) MHC class II tetramer staining: 1:100 dilution of PE-conjugated DR4/HA tetramer (DRB1*0401/HA $306-318$ [PKYVKQNTLKLAT]; kind gift from William Kwok, NIH Tetramer Facility, Emory University, Atlanta, Georgia, USA) or negative control tetramer (PE-conjugated DRB1*0401/GAD [NFIRMVISNPAAT] was added and incubated at $37^{\circ} \mathrm{C}$ for 30 minutes; (c) MHC class II tetramer staining: 1:125 dilution of PE-conjugated DR1/HA (DRB1*0101/HA 306-318 [PKYVKQNTLKLAT]; NIH Tetramer Facility, Virginia Mason Research Center) or negative control tetramer (PE-DRB1*0401/HA) was added and incubated at $37^{\circ} \mathrm{C}$ for 30 minutes. DRB $1 * 0401^{+}$-HA T cell clone HA136 was provided as a kind gift from JeanFrancois Fonteneau, Rockefeller University, New York, New York, USA, and was used as a positive control. After tetramer staining, cells were washed twice with medium, blocked for 15 minutes, and then incubated for another 15 minutes with 1:125 dilution of CD14-Alexa Fluor 700 (BD Biosciences Pharmingen) (all experiments) combined with anti-CD19-Alexa Fluor 700 (eBioscience) $(n=13$ experiments) (final 1:16 dilution with anti-CD14) along with 1:20 dilution of anti-CD8-Cy5 (CYTO-STAT/Coulter Clone; Beckman Coulter) for the MHC class I experiment or anti-CD4-FITC or anti-CD4-PE-Cy7 (Beckman Coulter) for the MHC class II experiments.

Additional cell-surface staining for CD45RO was performed in a subset using APC-conjugated anti-human CD45RO (1:10 dilution) or the appropriate isotype control (BD Biosciences - Pharmingen). In all experiments, tetramer staining was followed by staining with DAPI $(1 \mu \mathrm{g} / \mathrm{ml})$ (Molecular Probes) as an indicator of cell viability. In all experiments, an additional negative control was provided by simultaneously staining cells obtained from a vaccinated adult and/or cord blood from a newborn of a vaccinated adult who possessed a different HLA type. Stained cells were immediately analyzed by flow cytometry using a FACSCalibur or LSR II (BD Biosciences). Results were analyzed using FlowJo software (version 8.3.3; Tree Star Inc.). Dead cells and monocytes were excluded first based on the staining of DAPI and anti-CD14. The frequency of DR4/HA, DR1/HA or A2/MP tetramer staining was determined by dividing the gated live, $\mathrm{CD} 14^{-}$, tetramer-positive events by the number of gated, live CD $14^{-} \mathrm{CD} 4^{+}$or $\mathrm{CD} 14^{-} \mathrm{CD} 8^{+}$events.

Proliferation assay. Freshly prepared CBMCs or maternal PBMCs $\left(2 \times 10^{5} /\right.$ well $)$ were cultured in triplicate for 5 days with phytohemagglutinin as a positive control $(10 \mu \mathrm{g} / \mathrm{ml})$ (Sigma-Aldrich), dialyzed influenza vaccine $(0.25 \mu \mathrm{g} / \mathrm{ml})$, tetanus toxoid (1:500 dilution) (Wyeth), dialyzed JE vaccine $(0.25 \mu \mathrm{g} / \mathrm{ml})$, or no antigen. Proliferation was measured by thymidine incorporation on the fifth day. Antigen-specific proliferative responses were expressed as a stimulation index (SI), calculated by dividing the average counts per minute in antigencontaining wells by counts per minute in wells not containing antigen (5).

CSFE tracking dye, in vitro proliferation, and tetramer staining. Thawed CBMCs were resuspended in prewarmed RPMI 1640 medium at a final concentration of $1 \times 10^{6}$ cells $/ \mathrm{ml}$ and incubated at $37^{\circ} \mathrm{C}$ for 30 minutes. Cells were then incubated with $5 \mu \mathrm{M}$ 5- and 6-CFSE (Molecular Probes) in RPMI 1640 medium at $37^{\circ} \mathrm{C}$ for 15 minutes. Immediately after the incubation, cells were centrifuged and resuspended in prewarmed medium and then incubated another 30 minutes to ensure modification of CFSE. Cells were washed twice with RPMI medium and cultured in medium (Iscove's medium
[Mediatech Inc.] with $15 \%$ human serum) in the presence of dialyzed influenza virus vaccine, Fluzone $(0.25 \mu \mathrm{g} / \mathrm{ml})$, or inactivated JE virus vaccine $(0.25 \mu \mathrm{g} / \mathrm{ml})$. Anti-CD3 (10 ng/ml; BD Biosciences) was added to the culture. After 7 days of culture, CFSE-labeled cells were washed twice with cold PBS and stained with DR4/HA or DR1/HA tetramer and subsequently with anti-CD4, anti-CD14, anti-CD19 antibodies. Cells were acquired by an LSR II after being stained with dead cell marker DAPI $(1 \mu \mathrm{g} / \mathrm{ml})$ (Molecular Probes) and analyzed with FlowJo software (version 8.3.3; Tree Star Inc.).

Statistics. Samples were excluded from analyses if vaccination occurred more than 34 weeks prior to birth $(n=1)$ or if the mother suffered documented influenza infection despite vaccination $(n=1)$. Anti-Fluzone and anti-MP IgM and IgG antibody levels were calculated by subtracting the average OD measured in the absence of coated antigen from the average OD measured from wells coated with Fluzone, MP, or JE. Negative values (i.e., average measured $\mathrm{OD}$ following coating with antigen was lower than in the absence of antigen) were recorded as 0 . In cases when the denominator of the ratio was 0 (e.g., when prevaccination $\mathrm{OD}$ was 0 ), values were recoded as the prevaccine background (OD in non-antigen-coated well). A positive maternal anti-IgM response was defined as an average postvaccination OD 2-fold greater than the average prevaccination OD. A positive cord blood anti-IgM response to influenza proteins was defined as an average cord blood OD that was 2 -fold greater than the mean OD measured from the negative control, unvaccinated cohort described above. ODs from each IgG measurement were normalized as 1:10,000 dilutions.

Positive tetramer staining was defined as the detection of live, CD14$\mathrm{CD}^{+}$, or $\mathrm{CD}^{+}$cells that stained in the presence of the A2/MP, DR4/HA, or DR1/HA tetramer, after subtracting summed background staining indicated by both the negative control tetramer and by cells derived from an HLA A*0201-, DRB1*0101-, or DRB1*0401-individual stained with the A2/MP, DR1/HA, or DR4/HA tetramer, respectively.

Nonparametric statistical analyses of the antibodies and percentage tetramer staining were performed using the Mann Whitney $U$ test across groups (e.g., maternal versus cord blood levels) and Spearman's rho for correlations (e.g., IgM versus IgG levels) using VassarStats online statistical computation software (http://faculty.vassar.edu/lowry/VassarStats.html). Data are reported as mean \pm standard error (SEM) unless otherwise noted. Statistical significance was specified as a 2 -sided $P$ value of 0.05 or less.

\section{Acknowledgments}

This work was supported by Asthma and Allergic Diseases Research Center (grant 1 P01 AI50514), General Clinical Research Center (grant RR00645), NIH Tetramer Facility, the National Institute of Environmental Health Sciences (grants P01 ES09600, 5 RO1 ES08977, P30 ES009089), and the US Environmental Protection Agency (grant R827027). The authors are grateful to Tony Brown, Diana Blythe, Stephen Canfield, Raphael Clynes, Manisha Ballaney, Megan Williams, Vivek Iyer, Alina Johnson, Sullafa Kadura, Ji Yong Kong, Fangjun Liu, Jinming Liu, Claudia Maria, Vijay Mukhija, Judy Nam, Yoshiko Ogawa, James Spencer, Danila Valmori, and Veronika Vasquez for assisting in the conduction of this study. The following tetramers were obtained from the Virginia Mason Research Center through the NIH Tetramer Facility: DR1/HA, DR4/HA.

Received for publication June 21, 2006, and accepted in revised form April 9, 2007.

Address correspondence to: Rachel L. Miller, PH8C, Columbia University College of Physicians and Surgeons, 630 W. 168th Street, New York, New York 10032, USA. Phone: (212) 305-7759; Fax: (212) 305-2277; E-mail: rlm14@columbia.edu. 
1. Kondo, N., et al. 1992. Cord blood lymphocyte responses to food antigens for the prediction of allergic disorders. Arch. Dis. Child. 67:1003-1007.

2. Piastra, M., et al. 1994. Cord blood mononuclear cell responsiveness to beta-lactoglobulin: T-cell activity in "atopy-prone" and "non-atopy-prone" newborns. Int. Arch. Allergy Immunol. 104:358-365.

3. Piccinni, M.P., et al. 1993. Aeroallergen sensitization can occur during fetal life. Int. Arch. Allergy Immunol. 102:301-303.

4. Holt, P.G., et al. 1995. T-cell "priming" against environmental allergens in human neonates: sequential deletion of food antigen reactivity during infancy with concomitant expansion of responses to ubiquitous inhalant allergens. Pediatr. Allergy Immunol. 6:85-90.

5. Miller, R.L., et al. 2001. Prenatal exposure, maternal sensitization, and sensitization in utero to indoor allergens in an inner-city cohort. Am. J. Respir. Crit. Care Med. 164:995-1001.

6. Kondo, N., et al. 1998. Reduced interferon gamma production by antigen-stimulated cord blood mononuclear cells is a risk factor of allergic disorders-6 year follow-up study. Clin. Exp. Allergy. 28:1340-1344.

7. Prescott, S.L., King, B., Strong, T.L., and Holt, P.G. 2003. The value of perinatal immune responses in predicting allergic disease at 6 years of age. Allergy. 58:1187-1194.

8. Blumer, N., Herz, U., Wegmann, M., and Renz, H. 2005. Prenatal lipopolysaccharide-exposure prevents allergic sensitization and airway inflammation, but not airway responsiveness in a murine model of experimental asthma. Clin. Exp. Allergy. 35:397-402.

9. Liu, C.A., et al. 2003. Prenatal prediction of infant atopy by maternal but not paternal total IgE levels. J. Allergy Clin. Immunol. 112:899-904.

10. Platts-Mills, T.A.E., and Woodfolk, J.A. 2000. Cord blood proliferative responses to inhaled allergens: is there a phenomenon? J. Allergy Clin. Immunol. 106:441-443.

11. Thornton, C.A., et al. 2004. Functional maturation of CD4+CD25+CDLA4+CD45RA+ T regulatory cells in human neonatal $\mathrm{T}$ cell responses to environmental antigens/allergens. J. Immunol. 173:3084-3092.

12. Yabuhara, A., et al. 1997. TH2-polarized immunological memory to inhalant allergens in atopics is established during infancy and early childhood. Clin. Exp. Allergy. 27:1261-1269.

13. Ramage, J.M., Young, J.L., Goodall, J.C., and Gaston, J.S.H. 1999. T cell responses to heat-shock protein 60: differential responses by CD4+ T cell subsets according to their expression of CD45 isotypes. J. Immunol. 162:704-710.

14. Young, J.L., Daser, A., and Beverley, P.C. 1995. In vitro proliferative responses of human peripheral blood mononuclear cells to non-recall antigens. J. Immunol. Methods. 182:177-184.

15. Hobeika, A.C., et al. 2005. Enumerating antigenspecific T-cell responses in peripheral blood: a comparison of peptide MHC tetramer, ELISpot, and intracellular cytokine analysis. J. Immunother. 28:63-72.

16. Altman, J.D., et al. 1996. Phenotypic analysis of antigen-specific T lymphocytes. Science. 274:94-96.

17. Dunbar, P.R., et al. 1998. Direct isolation, phenotyping and cloning of low-frequency antigenspecific cytotoxic $\mathrm{T}$ lymphocytes from peripheral blood. Curr. Biol. 8:413-416.

18. Wu, Y., et al. 2004. Frequencies of epitope-specific cytotoxic $\mathrm{T}$ lymphocytes in active chronic viral hepatitis B infection by using MHC class I peptide tetramers. Immunol. Lett. 92:253-258.

19. McCloskey, T.W., Haridas, V., and Pahwa, R. 2001 Human immunodeficiency virus gag and pol-specific CD8 T cells in perinatal HIV infection. Cytometry. 46:265-270.

20. Marchant, A., et al. 2003. Mature CD8 ${ }^{+}$T lymphocyte response to viral infection during fetal life. J. Clin. Invest. 111:1747-1755. doi:10.1172/ JCI200317470.

21. Vanderbeeken, Y., Sarfati, M., Bose, R., and Delespesse, G. 1985. In utero immunization of the fetus to tetanus by maternal vaccination during pregnancy. Am. J. Reprod. Immunol. Microbiol. 8:39-42.

22. Gill, T.J.I., et al. 1983. Transplacental immunization of the human fetus to tetanus by immunization of the mother. J. Clin. Invest. 72:987-996.

23. Englund, J.A., et al. 1993. Maternal immunization with influenza or tetanus toxoid vaccine for passive antibody protection in young infants. J. Infect. Dis. 168:647-656

24. Centers for Disease Control and Prevention (CDC). 2005. Update: influenza vaccine supply and recommendations for prioritization during the 2005-06 influenza season. MMWR Morb. Mortal. Wkly. Rep. 54:850

25. Browning, M., and Krausa, P. 1996. Genetic diversity of HLA-A2: evolutionary and functional significance. Immunol. Today. 17:165-170.

26. Cao, K., et al. 2001. Analysis of the frequencies of HLA-A, B, and C alleles and haplotypes in the five major ethnic groups of the United States reveals high levels of diversity in these loci and contrasting distribution patterns in these populations. Hum. Immunol. 62:1009-1030.

27. Ellis, J.M., et al. 2000. Frequencies of HLA-A2 alleles in five U.S. population groups. Hum. Immunol. 61:334-340.

28. Mori, M., Beatty, P.G., Graves, M., Boucher, K.M., and Milford, E.L. 1997. HLA gene and haplotype frequencies in the North American population. Transplantation. 64:1017-1027.

29. Reed, E., Lupu, F., McManus, P., Seigle, R., and Sucio-Foca, N. 1992. Population and family studies of HLA-DR4 by use of oligonucleotide typing. Tissue Antigens. 39:266-271.

30. Sumaya, C.V., and Gibbs, R.S. 1979. Immunization of pregnant women with influenza A/New Jersey/76 virus vaccine: reactogenicity and immunogenicity in mother and infant. J. Infect. Dis. 140:141-146.

31. Baker, C.J., et al. 1988. Immunization of pregnant women with a polysaccharide vaccine of group $b$ streptococcus. N. Engl. J. Med. 319:1180-1185.

32. Firan, M., et al. 2001. The MHC class I-related receptor, FcRn, plays an essential role in the maternofetal transfer of gamma-globulin in humans. Int. Immunol. 13:993-1002.

33. Siegrist, C.A. 2001. Neonatal and early life vaccinology. Vaccine. 19:3331-3346.

34. Hermann, E., et al. 2002. Human fetuses are able to mount an adultlike CD8 T-cell response. Blood. 100:2153-2158.

35. Givan, A.L., et al. 2004. Use of cell-tracking dyes to determine proliferation precursor frequencies of antigen-specific T cells. Methods Mol. Biol. 263:109-124

36. Turner, S.J., Cross, R., Xie, W., and Doherty, P.C. 2001. Concurrent naive and memory CD8 (+) T cell response to an influenza A virus. J. Immunol. 167:2753-2758.

37. Hoffmann, T.K., et al. 2000. Competition of peptideMHC I tetrameric complexes with anti-CD3 provides evidence for specificity of peptide binding to the TCR complex. Cytometry. 41:321-328.

38. Danke, N.A., and Kwok, W.W. 2003. HLA class IIrestricted CD4+ $\mathrm{T}$ cell responses directed against influenza viral antigens postinfluenza vaccination. J. Immunol. 171:3163-3169.

39. Speiser, D.E., et al. 2004. Ex vivo analysis of human antigen-specific CD8+ T-cell responses: quality assessment of fluorescent HLA-A2 multimer and interferon-gamma ELISPOT assays for patient immune monitoring. J. Immunother. 27:298-308.

40. Deng, Y., Jing, Y., Campbell, A.E., and Gravenstein, S. 2004. Age-related impaired type I T cell responses to influenza: reduced activation ex vivo, decreased expansion in CTL culture in vitro, and blunted response to influenza vaccination in vivo in the elderly. J. Immunol. 172:3437-3446.

41. Haining, W.N., et al. 2004. Measuring T cell immunity to influenza vaccination in children after haemopoietic stem cell transplantation. Br. J. Haematol. 127:322-325.

42. Avetisyan, G., Ragnavolgyi, E., Toth, G.T., Hassan, M., and Ljungman, P. 2005. Cell-mediated immune responses to influenza vaccination in healthy volunteers and allogeneic stem cell transplant recipients. Bone Marrow Transplant. 36:411-415.

43. Hassan, J., and Reen, D.J. 2001. Human recent thymic emigrants - identification, expansion and survival characteristics. J. Immunol. 167:1970-1976.

44. DeVries, E., et al. 2000. Longitudinal survey of lymphocte subpopulations in the first year of life. Pediatr. Res. 47:528-537.

45. Albrecht, P., Ennis, F.A., Saltzman, E.J., and Krugman, S. 1997. Persistence of maternal antibody in infants beyond 12 months: mechanism of measles vaccine failure. J. Pediatr. 91:715-718.

46. Fiore, A.E., et al. 2003. Hepatitis A vaccination of infants: effect of maternal antibody status on antibody persistence and response to a booster dose. Pediatr. Infect. Dis. J. 22:354-359.

47. Manickan, E. 1997. DNA immunization of neonates induces immunity despite the presence of maternal antibody. J. Clin. Invest. 100:2371-2375.

48. Radu, D.L., et al. 2001. Effect of maternal antibodies on influenza virus-specific immune response elicited by inactivated virus and naked DNA. Scand. J. Immunol. 53:475-482.

49. Reuman, P.D., Paganini, C.M., Ayoub, E.M., and Small, P.A. 1983. Maternal-infant transfer of influenza-specific immunity in the mouse. J. Immunol. 130:932-936.

50. Mallone, R., and Nepom, G.T. 2004. MHC class II tetramers and the pursuit of antigen-specific $T$ cells: define, deviate, delete. Clin. Immunol. 110:232-242.

51. Shi, S.-R., et al. 2002. DNA extraction from archival formalin-fixed, paraffin-embedded tissue sections based on the antigen retrieval principle: heating under the influence of pH. J. Histochem. Cytochem. 50:1005-1011.

52. Zetterquist, H., and Olerup, O. 1992. Identification of the HLA-DRB $1 * 04,-\mathrm{DRB} 1 * 07$, and -DRB1*09 alleles by PCR amplification with sequence-specific primers (PCR-SSP) in 2 hours. Hum. Immunol. 34:64-74.

53. Krausa, P., et al. 1995. Genetic polymorphism within HLA-A*02: significant allelic variation revealed in different populations. Tissue Antigens. 45:223-231.

54. Buyse, I., et al. 1993. Rapid DNA typing of class II HLA antigens using the polymerase chain reaction and reverse dot blot hybridization. Tissue Antigens. 41:1-14.

55. Krausa, P., and Browning, M.J. 1996. A comprehensive PCR-SSP typing system for identification of HLA-A locus alleles. Tissue Antigens. 47:237-244. 